\title{
Optical tracking of picosecond coherent phonon pulse focusing inside a sub-micron object
}

\author{
Thomas Dehoux ${ }^{1}$, Kenichi Ishikawa ${ }^{2}$, Paul H Otsuka ${ }^{2}$, Motonobu Tomoda ${ }^{2}$, Osamu Matsuda ${ }^{2}$, \\ Masazumi Fujiwara ${ }^{3,4}$, Shigeki Takeuchi ${ }^{3,5}$, Istvan A Veres ${ }^{6}$, Vitalyi E Gusev ${ }^{7}$ and Oliver B Wright ${ }^{2}$
}

By means of an ultrafast optical technique, we track focused gigahertz coherent phonon pulses in objects down to sub-micron in size. Infrared light pulses illuminating the surface of a single metal-coated silica fibre generate longitudinal-phonon wave packets. Reflection of visible probe light pulses from the fibre surface allows the vibrational modes of the fibre to be detected, and Brillouin optical scattering of partially transmitted light pulses allows the acoustic wavefronts inside the transparent fibre to be continuously monitored. We thereby probe acoustic focusing in the time domain resulting from generation at the curved fibre surface. An analytical model, supported by three-dimensional simulations, suggests that we have followed the focusing of the acoustic beam down to a $\sim 150-\mathrm{nm}$ diameter waist inside the fibre. This work significantly narrows the lateral resolution for focusing of picosecond acoustic pulses, normally limited by the diffraction limit of focused optical pulses to $\sim 1 \mu \mathrm{m}$, and thereby opens up a new range of possibilities including nanoscale acoustic microscopy and nanoscale computed tomography.

Light: Science \& Applications (2016) 5, e16082; doi:10.1038/lsa.2016.82; published online 20 May 2016

Keywords: acoustic-optic; fibre; optical scattering; picosecond; ultrasonics

\section{INTRODUCTION}

Confining light and high-frequency sound waves in micron- to nanometre-scale structures, such as planar microcavities ${ }^{1,2}$, microtori $^{3}$, micro-ladders ${ }^{4}$, photonic crystal fibres ${ }^{5}$, nanorings ${ }^{6}$, nanovoids ${ }^{7}$ or nanoparticles ${ }^{8}$ promises enhanced interaction and control of light, with wide-ranging applications in cooling, sensing, optical modulation, frequency conversion or amplification. Time-resolved studies are of particular interest $\mathrm{t}^{1,2,6-8}$ as they yield information on the progression of acoustic pulses through the structure, thus allowing the probing of its interior. Picosecond acoustic pulses passing through microcavities or quantum wells ${ }^{1,9}$ have revealed, for example, the dynamics of carrier quantum-state coupling to resonant photons or polaritons, allowing new avenues for acousto-optic modulation to be explored.

Viewing the success of megahertz $(\mathrm{MHz})$ acoustic pulses in resolving millimetre-scale structures and subsequent extension to gigahertz $(\mathrm{GHz})$ imaging on micron scales ${ }^{10}$, it is natural to ask the question: can three-dimensional (3D) focused acoustic probing of a sub-micron structure be carried out? Steps in this direction have been made by wavefront imaging of $\mathrm{GHz}$ acoustic waves ${ }^{11,12}$ or by measuring acoustic echoes in microscopic spheres and cylinders ${ }^{13,14}$, as well as free-standing nanowires ${ }^{15-18}$, but it has not been possible to continuously follow the passage of focused acoustic waves in submicron-sized 3D objects, and thereby probe spatially resolved acoustic-optic interactions inside them in real time. In this paper, we combine the technique of generating coherent acoustic waves on curved surfaces with a time-domain Brillouin scattering technique to demonstrate the continuous optical tracking of focused $\mathrm{GHz}$ coherent phonon pulses in single silica fibres of radius down to $\sim 400 \mathrm{~nm}$ as well as the detection of their $\mathrm{GHz}$ vibrational modes. An analytical model, supported by 3D simulations, suggests that we have focused a $\sim 40-\mathrm{GHz}$ acoustic beam to a spot $\sim 150 \mathrm{~nm}$ in diameter.

\section{MATERIALS AND METHODS}

The sample is a tapered silica fibre of refractive index $n=1.47$ made by drawing a heated sample ${ }^{19}$, with core and cladding diameters 4.4 and $125 \mu \mathrm{m}$, respectively, in the undrawn fibre (single-mode fibre, Nufern $780-\mathrm{HP}$ ). The outer fibre radius increases gradually from $390 \mathrm{~nm}$ in the middle of the fibre length to several micrometres over a fibre length of $\sim 0.1 \mathrm{~m}$. For the purposes of local acoustic measurements on different parts of the fibre, the effect of the taper is negligible. An aluminium film of thickness $d=30 \mathrm{~nm}$ is sputtered on one side of the fibre for acoustic transduction. The difference in optical and mechanical properties of the core and cladding can be neglected.

We use an optical pump-probe technique, shown schematically in Figure 1a. Trains of optical pulses (wavelength $800 \mathrm{~nm}$, pulse duration $200 \mathrm{fs}$, energy $0.1 \mathrm{~nJ}$ and repetition frequency $81 \mathrm{MHz}$ ) are used as a pump. They are focused onto the metal-coated side of the fibre at normal incidence through a $100 \times$ objective lens (numerical

\footnotetext{
${ }^{1}$ Institut Lumière Matière, UMR5306, Université Lyon 1-CNRS, Université de Lyon, 69622 Villeurbanne, France; ${ }^{2}$ Division of Applied Physics, Faculty of Engineering, Hokkaido University, Sapporo 060-8628, Japan; ${ }^{3}$ Research Institute for Electronic Science, Hokkaido University, Sapporo 001-0020, Japan; ${ }^{4}$ School of Science and Technology, Kwansei Gakuin University, Sanda, Hyogo 669-1337, Japan; ${ }^{5}$ Department of Electronic Science and Engineering, Kyoto University, Kyoto 615-8510, Japan; 6 Research Centre for NonDestructive Testing GmbH, Altenberger Strasse 69, A-4040 Linz, Austria and ${ }^{7}$ Laboratoire d'Acoustique de I'Université, du Maine, Le Mans 72085 , France Correspondence: OB Wright, Email: olly@eng.hokudai.ac.jp

Received 29 September 2015; revised 18 January 2016; accepted 22 January 2016; accepted article preview online 22 February 2016
} 
aperture $=0.95)$ to a circular Gaussian spot of diameter $2 W_{\mathrm{pp}} \approx 1.6 \mu \mathrm{m}$ (at $1 / e^{2}$ intensity), with the optical polarization parallel to the fibre axis. The pump beam is chopped at $1 \mathrm{MHz}$ for lock-in detection. Each pump pulse produces a transient lattice temperature rise of $\sim 200 \mathrm{~K}$ (there is also a steady-state temperature rise of $\sim 50 \mathrm{~K}$, with heat loss mainly occurring in the $\mathrm{Al} \mathrm{film)} \mathrm{and} \mathrm{a} \mathrm{thermoelastic} \mathrm{expansion} \mathrm{of} \mathrm{the}$ $\mathrm{Al}$ film that launches longitudinal coherent phonon wave packets and triggers fibre vibrations. The small acoustic-strain-pulse reflection coefficient $\sim 0.13$ at the $\mathrm{Al} / \mathrm{SiO}_{2}$ interface (see Supplementary Information for physical properties) ensures efficient transmission to the fibre. A broadband excitation spectrum containing frequencies up to $\sim 150 \mathrm{GHz}$ and with strain amplitudes $\sim 5 \times 10^{-3}$ is obtained.

For illustration, the calculated bipolar spatial form of the generated coherent phonon strain pulse initially launched into the fibre is shown in the inset of Figure 2. This strain pulse shape is obtained by considering the effect of the ultrafast diffusion of optically excited electrons in the $\mathrm{Al}$ film with a one-dimensional two-temperature model ${ }^{20-23}$. (Acoustic diffraction in $3 \mathrm{D}$ can be neglected for the
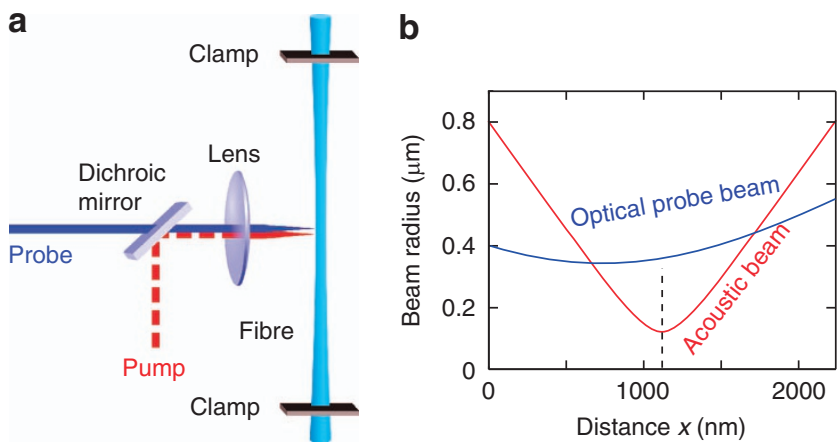

Figure 1 Experimental setup, and the acoustic and optical profiles for fibre radius $R_{f}=1150 \mathrm{~nm}$ based on Gaussian beam theory. (a) Sample and setup. (b) Theoretical optical probe (blue) and acoustic-strain (red) $1 / e^{2}$ beam radii ( $w_{E}(x)$ and $w_{A}(x)$, respectively) as a function of propagation distance $x$ (over the region from the front surface of the fibre to the back) calculated from Gaussian-beam theory for a fibre of radius $R_{f}=1150 \mathrm{~nm}$. (The dashed line indicates the position of the centre of the fibre.)

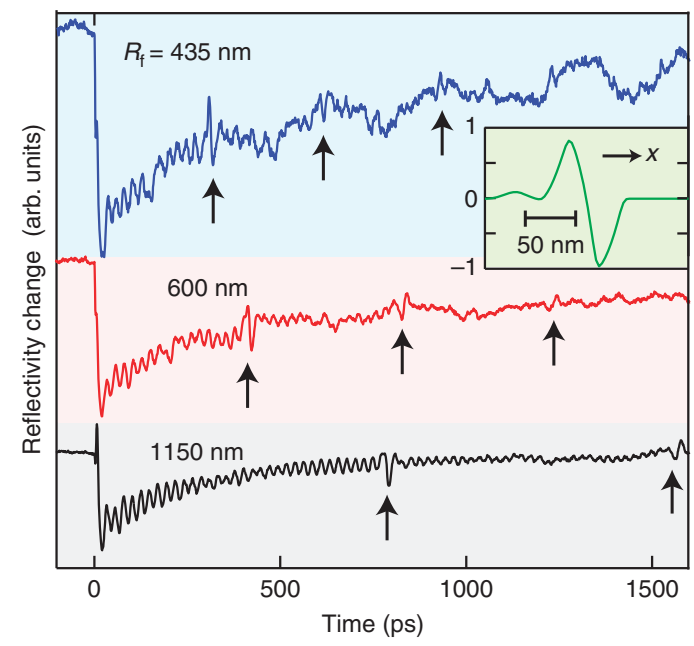

Figure 2 Optical reflectivity changes measured at three different locations on the fibre. Upward arrows indicate acoustic echoes. Inset: calculated strain pulse in the fibre just after leaving the Al film, where $x$ is the radial coordinate measured in the direction from the excited surface to the centre of the fibre. propagation distances under consideration.) The nonlinear equations are solved numerically by the finite-difference time-domain method to obtain the spatiotemporal evolution of the electron and lattice temperatures (see parameters in Supplementary Information). The lattice temperature change generates the strain by thermal expansion, and the longitudinal strain field is calculated before transmission to the silica. Standard equations for acoustic-strain transmission then yield the spatial strain-pulse shape in the silica. The slight bump on the trailing (left-hand) side of the strain pulse is caused by acoustic reverberation inside the $\mathrm{Al}$ film due to the above-mentioned small strain reflection coefficient at the $\mathrm{Al} / \mathrm{SiO}_{2}$ interface. Nonlinear effects arising from the two-temperature model are significant in this calculation, leading to a rounding effect on the acoustic pulse shape. Thermal conduction to the relatively poorly conducting silica is ignored.

The optical reflectivity change $\delta R(t)$ is measured as a function of pump-probe time delay $t$ with frequency-doubled circularly polarized probe pulses (wavelength $\lambda=400 \mathrm{~nm}$ and energy $40 \mathrm{pJ}$ ). The probe is focused coaxially with the pump to a spot of diameter $2 W_{\mathrm{pb}} \approx 0.8 \mu \mathrm{m}$ (at $1 / e^{2}$ intensity) on the fibre. In practice, optical alignment was achieved by searching for the maximum detected ultrafast probe-beam intensity modulation. Owing to the small radius of curvature of the fibre, the reflected probe beam is strongly divergent; we measure an up to $\sim 90 \%$ clipping of this beam by the finite apertures of the objective, allowing detection enhancement through a defocusing effect ${ }^{13}$. In addition, given the refractive indices of $\mathrm{Al}, 0.52+5.0 i$, and of silica, 1.47 , at $400 \mathrm{~nm}, \sim 5 \%$ of the probe-beam intensity penetrates through the $\mathrm{Al}$ coating 24 . This enables the continuous photoelastic probing of the acoustic amplitude inside the transparent fibre. The experimental technique is largely based on the standard methods of picosecond ultrasonics ${ }^{25-27}$. However, probing the passage of focused acoustic waves in sub-micron-sized $3 \mathrm{D}$ objects, such as our fibre sample, has never been attempted.

\section{RESULTS AND DISCUSSION}

\section{Time-domain results}

We plot $\delta R(t)$ in Figure 2 for three different points along the tapered fibre axis, corresponding to radii $R_{\mathrm{f}}=435,600$ and $1150 \mathrm{~nm}$. A sharp spike at $t=0$ arises from electron excitation and strain generation in the $\mathrm{Al}$ coating. This is followed by a longer-timescale overall thermal decay. Owing to generation on a curved surface, the fibre acts as a converging cylindrical lens and focuses the coherent longitudinal acoustic phonon pulses inside it. These pulses are transmitted into the fibre and are reflected back from the opposite surface; on returning to the $\mathrm{Al}$ layer, they produce echoes (indicated by the upward arrows in Figure 2). The radii $R_{\mathrm{f}}=v_{1} \Delta t / 4$ can be determined from the time interval $\Delta t$ between two successive echoes, using the known longitudinal sound velocity in silica, $v_{1}=5970 \mathrm{~m} \mathrm{~s}^{-1}$ (ref. 24). Brillouin oscillations $^{26,28,29}$ at frequency $f_{\mathrm{B}} \approx 44 \mathrm{GHz}$ are clearly observable in the time domain for all the investigated positions on the tapered fibre. At the time when the phonon pulse reaches the back of the fibre wall, the oscillations show an approximate symmetry point owing to the acoustic reflection, as discussed in further detail below. There are also evident lower frequency contributions to $\delta R$, particularly clear for the case of $R_{\mathrm{f}}=435 \mathrm{~nm}$. These arise from vibrational modes of the cylindrical cross section of the fibre.

\section{Vibrational modes and Brillouin scattering}

Figure 3 shows the normalized Fourier spectra of $\delta R(t)$, indicating the presence of several vibrational modes. We have selected modes (downward arrows in Figure 3) that are common to two or more 
radii at frequencies $f_{\mathrm{V}}=4.3,9.6$ and $24.1 \mathrm{GHz}$ for $R_{\mathrm{f}}=435 \mathrm{~nm}$, at 3.2 , 7.0 and $17.8 \mathrm{GHz}$ for $R_{\mathrm{f}}=600 \mathrm{~nm}$, and at $1.7 \mathrm{GHz}$ for $R_{\mathrm{f}}=1150 \mathrm{~nm}$. The product $f_{\mathrm{V}} R_{\mathrm{f}}$ is shown in the inset on a log scale as a function of $R_{\mathrm{f}}$. We compare these results with the frequency $f_{l}^{m}$ of vibrations with mode numbers $(m, l)$ obtained from the elastic wave equation ${ }^{30}$ for a homogeneous isotropic silica cylinder (horizontal dashed lines in the inset) using literature values (density $\rho=2.2 \mathrm{~g} \mathrm{~cm}^{-3}$ and sound velocities $v_{\mathrm{l}}=5970 \mathrm{~m} \mathrm{~s}^{-1}$ and $v_{\mathrm{t}}=3760 \mathrm{~m} \mathrm{~s}^{-1}$ (ref. 24)). The pumpbeam spot and investigated fibre diameters are of the same order of magnitude, so we neglect the effect of acoustic-wave propagation along the cylinder axis. We identify a breathing mode $(0,0)$, a whispering gallery mode $(1,2)$ and a higher-frequency mode that is not uniquely assignable owing to mode overlap. The vibrational bandwidths $(\sim 0.5 \mathrm{GHz})$ are largely determined by the finite time window $(1.5 \mathrm{~ns})$ for the data acquisition.

The Brillouin oscillations arise from the interference between the light scattered from the strain pulse inside the fibre and that reflected

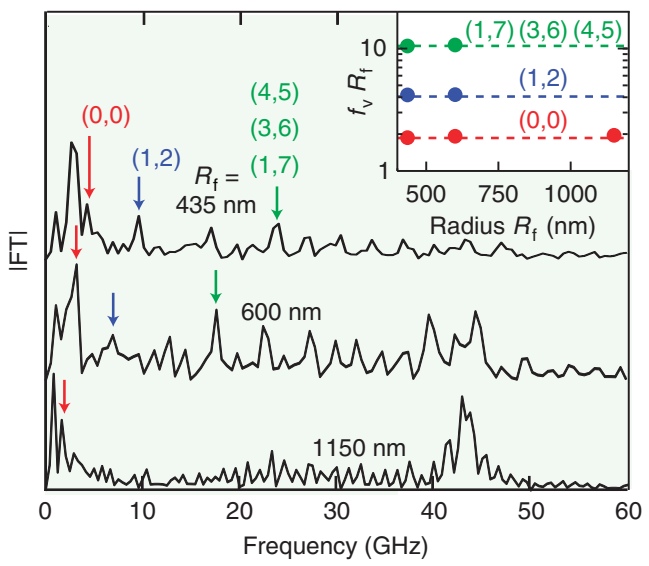

Figure 3 Experimental Fourier spectra. Fourier (modulus) spectra corresponding to Figure 2. Inset: measured (dots) and calculated (dashed lines) normalized vibrational frequencies on a log scale in $f_{v} R_{f}$ (measured in $\mathrm{km} \mathrm{s}^{-1}$ ) vs fibre radius.

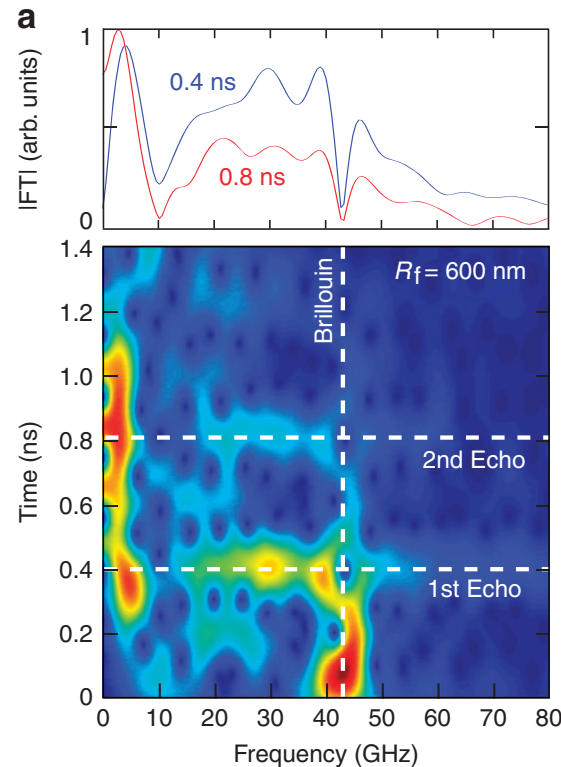

by the front surface of the fibre ${ }^{26,28}$. These oscillations are governed by optical scattering from phonons of wavelength $\lambda_{\mathrm{A}}=\lambda /(2 n)=136 \mathrm{~nm}$. The exact form of the strain pulse does not affect the evolution of the Brillouin oscillations, which occur at a quasi-single frequency. The measured frequency $f_{\mathrm{B}}=44 \mathrm{GHz}$ from the Fourier spectra of Figure 3 is in excellent agreement with the equation $f_{\mathrm{B}}=v_{1} / \lambda_{\mathrm{A}}$, which applies for normal optical incidence, using known values of $\lambda, n$ and $v_{1}$ (ref. 24). On increasing the fibre radius $R_{\mathrm{f}}, \delta R(t)$ exhibits an increasing contribution from Brillouin scattering as well as a decreasing contribution from the vibrational modes, the latter effect presumably arising because of the decreasing probe-beam clipping. No phase change in the Brillouin oscillations is observed as the phonon pulse passes through the acoustic focus near the centre of the fibre. Although a Gouy phase shift in the acoustic strain of $\pi / 2$ is predicted for transmission through a cylindrical focus ${ }^{31}$, the observed Brillouin oscillations in optical probe-beam reflectivity depend on the complex $3 \mathrm{D}$ photoelastic interaction, and there is no simple prediction for the phase shift. We describe below how the Brillouin oscillations can be used to continuously monitor the acoustic propagation inside the fibres.

\section{Time-frequency analysis}

To better resolve the different contributions to $\delta R(t)$, we perform a short-time Fourier transform (STFT) for the $R_{\mathrm{f}}=600$ and $1150 \mathrm{~nm}$ data. This analysis is not done for $R_{\mathrm{f}}=435 \mathrm{~nm}$ owing to the dominant contribution of the vibrational modes. The use of Hamming windows (of FWHM (full-width at half-maximum) 190 and 300 ps, respectively) minimizes the vibrational-mode contribution. Respective STFT images are shown in Figure $4 \mathrm{a}$ and $4 \mathrm{~b}$. In both cases, a greater amplitude is observed at $f_{\mathrm{B}} \approx 44 \mathrm{GHz}$ (vertical dashed lines), and also at 0.4 and $0.8 \mathrm{~ns}$ (lower horizontal dashed lines) at the times of the respective first echoes. Similar features for the second echoes are, respectively, observed at 0.8 and $1.6 \mathrm{~ns}$ (higher horizontal dashed lines). The dips in the response at these times $\sim 44 \mathrm{GHz}$ can be attributed to the phonon pulse transmission from the fibre through the Al film towards its mechanically free surface and back. At low

b
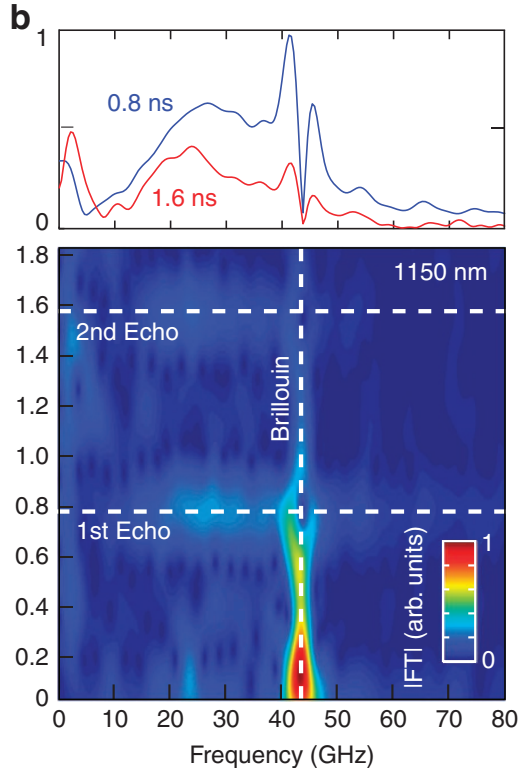

Figure 4 STFT data. STFT images of $\delta R$ and sections measured for (a) radius $R_{\mathrm{f}}=600 \mathrm{~nm}$ (Hamming window of FWHM $190 \mathrm{ps}$ ), and (b) $R_{\mathrm{f}}=1150 \mathrm{~nm}$ (window of $300 \mathrm{ps}$ ). 
frequencies for $R_{\mathrm{f}}=600 \mathrm{~nm}$, there also exists a strong, long-lived feature from the cylinder breathing mode.

For the case of $R_{\mathrm{f}}=600 \mathrm{~nm}$ (and $1150 \mathrm{~nm}$ ), we plot the spectra at the times of the first and second echoes at 0.4 and 0.8 ns (and 0.8 and $1.6 \mathrm{~ns}$ ), corresponding to sections along the horizontal lines in the STFT image in Figure $4 \mathrm{a}$ and $4 \mathrm{~b}$. The second-to-first echo ratio $r_{\mathrm{ac}}$ yields the frequency-dependent round-trip attenuation coefficient $\alpha=-\ln \left(r_{\mathrm{ac}}\right) /\left(4 R_{\mathrm{f}}\right)$, plotted in Figure $5 \mathrm{a}$. As a guide to the eye, a least-squares fit to $\alpha(f)=a f^{2}$, with $a$ as a constant, in the range $f=20-60 \mathrm{GHz}$ is also plotted for the case of $R_{\mathrm{f}}=1150 \mathrm{~nm}$. (Cylinder vibrational modes dominate below this range and measurement noise dominates above.) The near-quadratic frequency dependence and the value of $\alpha$ are in reasonable agreement with previous measurements of the ultrasonic attenuation in silica ${ }^{32}$.

\section{Photoelastic interaction in an equivalent lossless material}

In order to better follow the amplitude of the coherent phonon pulses through Brillouin oscillations, we removed the thermal relaxation and vibrational contributions to $\delta R(t)$ by subtraction of a polynomial fit to the background. We then obtained the envelope $E(t)$ of the Brillouin oscillations from the absolute value of the analytic signal ${ }^{33}$. $E(t)$ for the case of $R_{\mathrm{f}}=1150 \mathrm{~nm}$, for which the best signal-to-noise ratio was obtained, is shown in Figure 6a. The strain pulse first reaches the back of the fibre at $t_{2} \approx 0.4 \mathrm{~ns}$; it then returns to the front surface as the first echo at $\approx 0.8$ ns. Consider two times $t_{\mathrm{A}}<t_{2}$ and $t_{\mathrm{B}}=2 t_{2}-t_{\mathrm{A}}$ symmetrical with respect to their average, $t_{2}$, as shown in Figure 6a. The strain pulse is detected at the same distance $L=\left(t_{2}-t_{\mathrm{A}}\right) v_{1}$ from the back of the fibre at $t_{\mathrm{A}}$ and $t_{\mathrm{B}}$. The quantity $\ln \left[E\left(t_{\mathrm{A}}\right) / E\left(t_{\mathrm{B}}\right)\right] /(2 L)$ therefore yields the average ultrasonic attenuation at the Brillouin frequency, $\alpha_{\mathrm{B}}=\alpha\left(f_{\mathrm{B}}\right)$, over a region of length $2 L$. We plot $\alpha_{\mathrm{B}}$ vs $L$ in Figure $5 \mathrm{~b}$ for
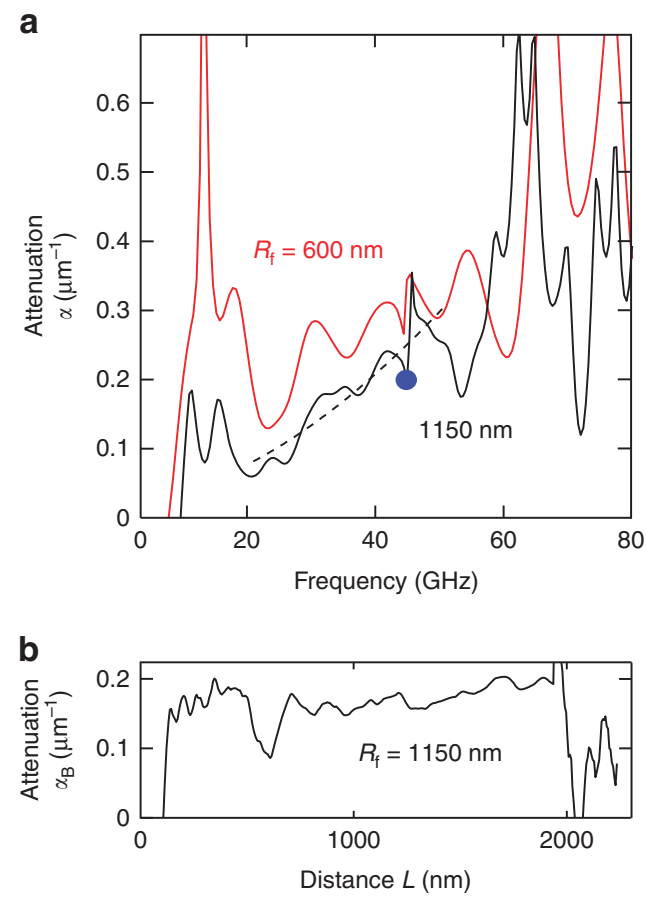

Figure 5 Experimental results for the ultrasonic attenuation. (a) Ultrasonic attenuation coefficient $\alpha(f)$ obtained from acoustic echoes for $R_{\mathrm{f}}=600$ and $1150 \mathrm{~nm}$. The dashed line is a quadratic fit to the $R_{\mathrm{f}}=1150 \mathrm{~nm}$ data. (b) Ultrasonic attenuation at $44 \mathrm{GHz}, \alpha_{\mathrm{B}}=\alpha\left(f_{\mathrm{B}}\right)$, vs distance $L$, obtained from Brillouin oscillations for the case of $R_{\mathrm{f}}=1150 \mathrm{~nm}$. The effective value derived from $\mathbf{b}$ is indicated by the dot in $\mathbf{a}$. the case of $R_{\mathrm{f}}=1150 \mathrm{~nm}$ using the same vertical scale as in Figure $5 \mathrm{a}$. The attenuation $\alpha_{\mathrm{B}} \sim 0.18 \mu \mathrm{m}^{-1}$ is largely constant (the dip at $600 \mathrm{~nm}$ arises from noise on $E\left(t_{\mathrm{B}}\right)$ ), showing good agreement with Figure $5 \mathrm{a}$ (as marked by a dot).

The envelope $E(t)$ is affected by both the spatial variation in the strainpulse amplitude and in the intensity of the optical probe. We define $E^{\prime}(x)=E(x) / \exp \left(-\alpha_{\mathrm{B}} x\right)$ to compensate for ultrasonic attenuation at $f_{\mathrm{B}}$, where in this formula $x$ is the radial coordinate folded around the symmetry point, which is at the back of the fibre at a distance $v_{1} t_{2}=2 R_{\mathrm{f}}$ from the front surface (the latter position being at $x=0$ ). Figure $6 \mathrm{~b}$ shows a plot of $E^{\prime}$ vs the distance $x$. $E^{\prime}$ represents the amplitude of the photoelastic interaction in an equivalent lossless material as the strain pulse propagates from the front to the back of the fibre (lower blue curve) and from the back to the front (upper red curve). The curves approximately superimpose, as expected, as losses have been eliminated. For $x \geqslant 1700 \mathrm{~nm}$, optical reflections from the back of the fibre modify the observed spatial variation of $E^{\prime}$. We therefore restrict our attention to the region $x \lesssim 1700 \mathrm{~nm}$. Over this region, $E^{\prime}$ shows a monotonic decrease. In the next section, we provide an analytical background to these observations.

\section{Discussion}

Consider the representative case of $R_{\mathrm{f}}=1150 \mathrm{~nm}$. We assume that the optical beam divergence is small enough for the paraxial (that is, Gaussian) approach to beam propagation to be approximately valid ${ }^{34}$. Consider first the evolution of the optical probe beam of wavelength $\lambda=400 \mathrm{~nm}$. This beam is focused to a spot of radius $W_{\mathrm{pb}} \approx 0.4 \mu \mathrm{m}$ at $1 / e^{2}$ intensity at the fibre surface (that is, at $x=0$, where the positive $x$ direction points towards the centre of the fibre; Figure 1). We define $w_{E}(x)$ as the optical probe-beam radius in air at $1 / e^{2}$ intensity:

$$
w_{E}(x)=W_{\mathrm{pb}}\left\{1+\left[\left(x-x_{E}^{0}\right) / x_{E}^{R}\right]^{2}\right\}^{1 / 2}
$$

where $x_{E}^{R}=\pi W_{\mathrm{pb}}^{2} / \lambda=1260 \mathrm{~nm}$ is the optical Rayleigh length in air. $x_{E}^{0}$ describes the position of the beam waist; for the incident beam, $x_{E}^{0}=0$. This beam is refracted at the fibre surface, and within the present approximation remains Gaussian inside the fibre with radii $w_{E y^{\prime}}$ and $w_{E z}{ }^{\prime}$ described by equations similar to Equation (1), where $y$ is the coordinate perpendicular to $x$ and to the fibre axis, and $z$ is the coordinate along the fibre axis. The laws of refraction at front side of the fibre, that is, at $x=0$, impose that the radii of curvature of the incident wavefront, $R_{E}$, and that of the wavefront refracted in the $y$ and $z$ directions, $R_{E y^{\prime}}$ and $R_{E z}{ }^{\prime}$, are such that $n / R_{E y}{ }^{\prime}(0)=1 / R_{E}(0)$
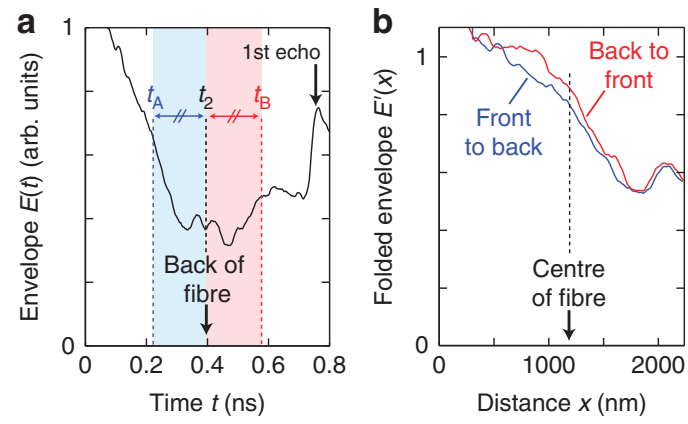

Figure 6 Results for the Brillouin-oscillation envelope and fits. Analysis of the data for the case of radius $R_{\mathrm{f}}=1150 \mathrm{~nm}$. (a) Normalized Brillouinoscillation envelope $E(t)$. (b) Equivalent plot of $E^{\prime}(x)$ for the lossless case as a function of the distance $x$ for propagation from the front surface of the fibre to the back and vice versa. 
$+(n-1) / R_{\mathrm{f}}, n / R_{E z}{ }^{\prime}(0)=1 / R_{E}(0)$, and that the radii of the beams are equal at this point, $w_{E}(0)=w_{E y}{ }^{\prime}(0)=w_{E z}{ }^{\prime}(0)^{35}$. The normalized $(x, y$, $z$ ) profile of the probe-beam intensity inside the fibre is given by:

$$
I(x, y, z)=\frac{2}{\pi w_{E y}^{\prime}(x) w_{E z}{ }^{\prime}(x)} \exp \left[-\frac{2 y^{2}}{{w_{E y}{ }^{\prime 2}(x)}^{2}}-\frac{2 z^{2}}{{w_{E z}{ }^{\prime 2}(x)}^{\prime}}\right]
$$

We plot the calculated Gaussian optical probe-beam radius inside the fibre, $w_{E y}{ }^{\prime}$, vs $x$ in Figure $1 \mathrm{~b}$ in blue. In the $z$ direction, the beam diverges slowly in a linear fashion. In the $y$ direction, the beam is weakly focused at position $x \approx 750 \mathrm{~nm}$ inside the fibre to a spot of $1 / e^{2}$ intensity radius $\sim 360 \mathrm{~nm}$.

Now consider the evolution of the acoustic beam for the case of $R_{\mathrm{f}}=1150 \mathrm{~nm}$. This beam should, for our acoustic wavelength $\lambda_{\mathrm{A}} \ll R_{\mathrm{f}}$, also approximately follow paraxial (Gaussian-beam) theory ${ }^{34,36}$. Its radius $w_{\mathrm{A}}(x)$ at $1 / e^{2}$ strain amplitude can be described by an equation similar to Equation (1), with a waist of $W_{\mathrm{ac}}$ (1/ $e^{2}$ radius). The radius of curvature of the acoustic beam, $R_{\mathrm{ac}}$, is equal to the radius of the fibre at the surface, $R_{\mathrm{ac}}(0)=R_{\mathrm{f}}$, and $w_{\mathrm{A}}$ is equal to the radius of the pump beam at the front side of the fibre, $w_{\mathrm{A}}(0)=W_{\mathrm{pp}}$. Since $W_{\mathrm{pp}} \gg \lambda_{\mathrm{A}}$, the location of the acoustic waist is very close to the centre of the fibre. (Here, for simplicity, we assume that acoustic diffraction, as well as acoustic propagation along the axis of the fibre, are negligible on the short timescales of our experiments, and that the acoustic beam radius $w_{\mathrm{A} z}$ along the $z$ direction is a constant determined by the optical pump-beam radius $W_{\mathrm{pp}} \approx 0.8 \mu \mathrm{m}$.) Under these approximations,

$$
w_{\mathrm{A}}(x)=W_{\mathrm{ac}}\left\{1+\left[\left(x-x_{\mathrm{A}}^{0}\right) / x_{\mathrm{A}}^{R}\right]^{2}\right\}^{1 / 2}
$$

where $\quad W_{\mathrm{ac}}=2 R_{\mathrm{f}} \lambda_{\mathrm{A}} /\left(\pi W_{\mathrm{pp}}\right) \approx 125 \mathrm{~nm}$, and $x_{\mathrm{A}}^{R}=\pi W_{\mathrm{ac}}^{2} /\left(2 \lambda_{\mathrm{A}}\right)=$ $179 \mathrm{~nm}$ is the acoustic Rayleigh length. $x_{\mathrm{A}}^{0}=R_{\mathrm{f}}$ under these approximations describes the position of the acoustic beam waist at the centre of the fibre. The strain pulse spans a distance $\sim 2 d=60 \mathrm{~nm} \ll R_{\mathrm{f}}$ along the $x$ axis (see inset of Figure 2a), thus, for the purposes of analysing Brillouin oscillations, one can approximate the temporal variation of the normalized $(x, y)$ profile of the longitudinal acoustic strain for $t<2 R_{\mathrm{f}} / v_{1}$ by the equation:

$$
\eta(x, y, t)=\delta\left(x-v_{1} t\right)\left[\frac{2}{\pi w_{\mathrm{A}}^{2}(x)}\right]^{1 / 4} \exp \left[-\frac{2 x^{2}}{w_{\mathrm{A}}^{2}(x)}\right]
$$

We plot the Gaussian acoustic-strain beam radius $w_{\mathrm{A}}$ vs $x$ calculated in this way in Figure $1 \mathrm{~b}$ for wavelength component $\lambda_{\mathrm{A}}=136 \mathrm{~nm}$ for the case of $R_{\mathrm{f}}=1150 \mathrm{~nm}$. The acoustic beam converges, focuses at the centre of the fibre to a spot of $1 / e^{2}$ radius for strain equal to $W_{\mathrm{ac}} \approx 125 \mathrm{~nm}$, which is similar in order to $\lambda_{\mathrm{A}}$, and then diverges.

This analytical approach can be validated by $3 \mathrm{D}$ finite-element simulations (see Supplementary Information for details). Figure 7a shows optical probe-beam simulations for an $R_{\mathrm{f}}=1150 \mathrm{~nm}$ uncoated (for simplicity) silica fibre, using the probe-beam parameters quoted above. (In practice, the fibre curvature and plasmonic excitation in the metal film will cause the pump-beam optical absorption over the fibre surface to depart to some extent from an exact Gaussian distribution, but we ignore these effects in this approximate analysis.) The expected divergence of the optical electric field $\left(E_{z}\right)$ distribution is clearly visible, and the simulated $1 / e^{2}$ beam radius for optical intensity (solid lines in Figure 7a) agrees reasonably well with Gaussian theory (dashed lines that ignore optical back reflections). The small wiggles in the simulated beam radius arise from sampling curved wavefronts along planes perpendicular to the $x$ direction (See Supplementary Information for further details).

Figure $7 \mathrm{~b}$ shows a single-frequency acoustic simulation of the longitudinal strain for the case of $R_{\mathrm{f}}=1150 \mathrm{~nm}$. (We have chosen
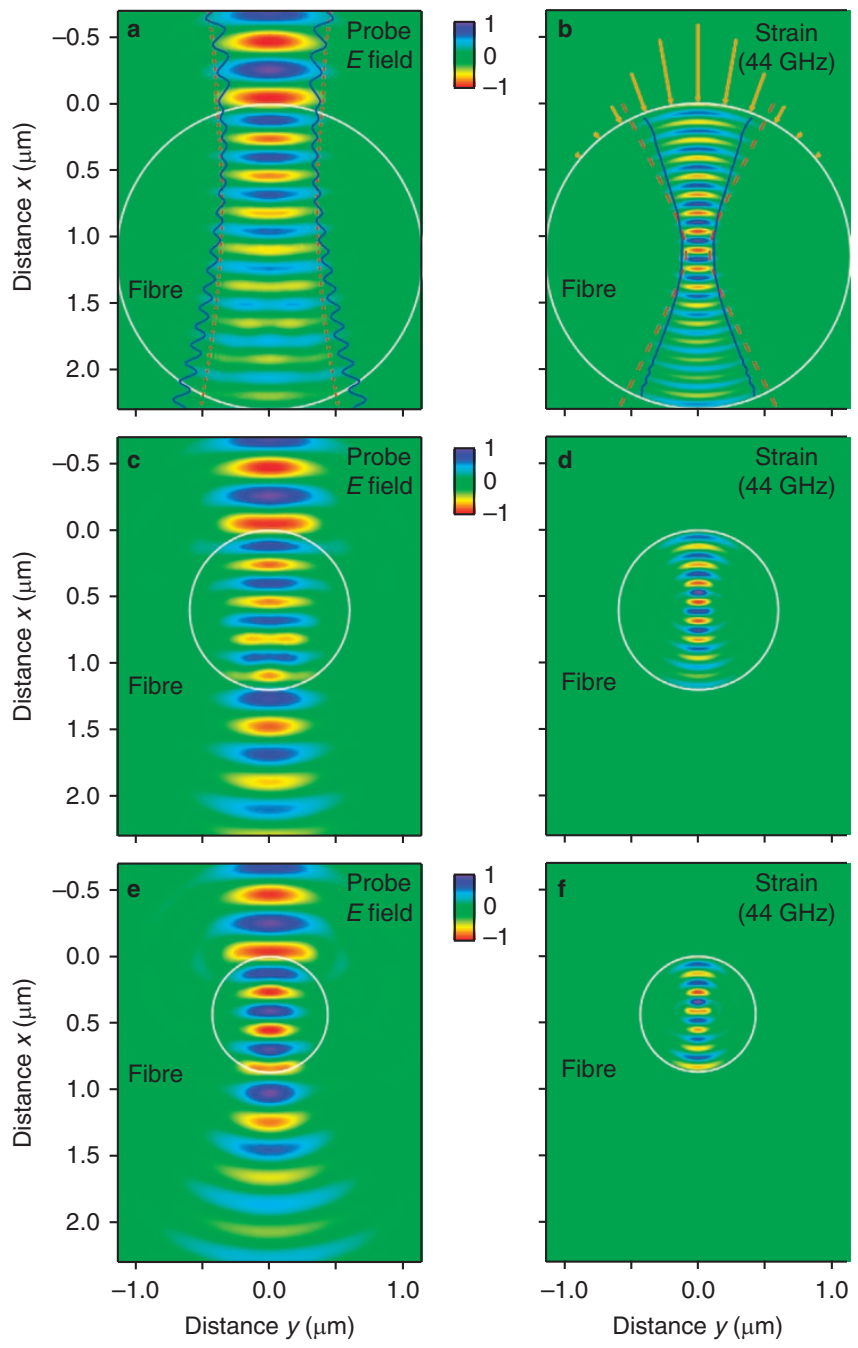

Figure 7 Optical and acoustic simulation results. (a, b) Simulated opticalprobe electric field amplitude $E_{z}$ and volumetric strain $\eta_{x x}+\eta_{y y}$ at $44 \mathrm{GHz}$, respectively, in an uncoated fibre of radius $R_{\mathrm{f}}=1150 \mathrm{~nm}$. Respective $1 / e^{2}$ intensity and volumetric-strain-squared $\left(\left(\eta_{x x}+\eta_{y y}\right)^{2}\right)$ beam radii $\left(w_{E}(x)\right.$ and $w_{\mathrm{A}}(x) / \sqrt{2}$, respectively) from the $3 \mathrm{D}$ numerical simulations (solid lines) and from Gaussian-beam theory (dashed lines) are superimposed. The arrows in $\mathbf{b}$ refer to the forces used for excitation. (c-f) Equivalent simulations for fibres of radius $R_{\mathrm{f}}=600$ and $435 \mathrm{~nm}$, respectively.

to plot strain-squared Gaussian profiles in Figure 7 by analogy with the optical-intensity Gaussian profiles in the same figure.) A sinusoidal force at $44 \mathrm{GHz}$ with a Gaussian spatial distribution on the fibre surface (over a $1 / e^{2}$ radius of $800 \mathrm{~nm}$ for strain) provides a reasonable approximation for the acoustic generation by the pump beam associated with the Brillouin oscillations. The $1 / e^{2}$ acoustic-strain-squared $\left(\left(\eta_{x x}+\eta_{y y}\right)^{2}\right)$ beam radius derived from the simulation (solid lines in Figure $7 \mathrm{~b}$ ) also agrees reasonably well with Gaussian theory (dashed lines that ignore acoustic back reflections). The small discrepancies arise because of the approximations inherent in the paraxial Gaussian-beam theory, the approximate strain distribution used on the fibre surface and the sampling of the strain field along curved wavefronts. (See Supplementary Information for further details.)

The simulated optical-probe (Figure 7c and 7e) and acoustic-strain (Figure $7 \mathrm{~d}$ and $7 \mathrm{f}$ ) fields for the two smaller-radii data sets are 
also shown in Figure 7, exhibiting similar behaviour to the case of $R_{\mathrm{f}}=1150 \mathrm{~nm}$ in each case. In particular, the acoustic spot sizes are comparable for all the fibre radii investigated. We did not include the Gaussian-beam theory for the smaller fibre radii because as the fibre radius becomes comparable to the wavelength or source sizes, this theory becomes less accurate.

The initial decrease and subsequent increase of the optical probebeam radius $w_{E}^{\prime}(x)$ with $x$ inside the fibre for the case of $R_{\mathrm{f}}=1150 \mathrm{~nm}$ and the acoustic focusing within the fibre govern the monotonic decrease of the lossless envelope $E^{\prime}(x)$, although a quantitative explanation is beyond the scope of this paper. The above analytical analysis, supported by the $3 \mathrm{D}$ simulations, suggests that for this fibre radius the acoustic beam is focused to a $1 / e^{2}$ radius beam waist of $W_{\mathrm{ac}} \approx 125 \mathrm{~nm}$ for the strain, that is, to a $150-\mathrm{nm}$ FWHM waist. This is similar to the acoustic spot sizes previously demonstrated in higherfrequency picosecond-ultrasonics-based experiments on layered semiconductor heterostructures (that is, a planar geometry not suitable for optical access from any direction $)^{9}$. In contrast, in the present experiments, the acoustic waves are focused inside a $3 \mathrm{D}$ micron- to sub-micron-sized structure close to a waist $\sim 150 \mathrm{~nm}$ in diameter, as is evident in the acoustic simulations for all the fibre radii investigated.

\section{CONCLUSIONS}

Picosecond ultrasonics is an all-optical technique for the generation and the detection of acoustic pulses of nanoscale wavelength by femtosecond laser pulses, with broad application to the nondestructive evaluation of nanostructured materials and fundamental research. However, this technique has been hampered for general application by the $\sim 1-\mu \mathrm{m}$ diffraction limit of focused light pulses, thus restricting the lateral resolution. Indeed, research on depth profiling using Brillouin scattering has not been able to overcome the optical diffraction limit for the lateral resolution ${ }^{29,37-39}$. The present research is an important step in removing this constraint. The choice of a sample with a curved surface in the form of a transparent silica fibre of radius as small as $\sim 400 \mathrm{~nm}$ allowed us to track the focusing of $\mathrm{GHz}$ coherent phonon wave packets in a 3D structure with outer dimensions down to sub-micron in size. As well as following these wave packets with Brillouin oscillations, we also accessed the vibrational resonances. An analytical model, supported by $3 \mathrm{D}$ simulations, suggests that the 44-GHz Brillouin component of the acoustic beam is focused to a spot $\sim 150 \mathrm{~nm}$ in diameter.

This technique holds great promise for future developments. The use of shorter-wavelength optical probe light or a higher refractiveindex medium would allow higher acoustic frequencies to be accessed, and to the production and monitoring of yet smaller acoustic spot sizes. This ability to monitor tight acoustic focusing within sub-micron transparent objects should allow the design of acoustic transducers for nanoscale imaging applications in cellular biology or nanotechnology. In particular, from a series of optical pump and probe measurements at different angles ${ }^{40}$ around sub-micron cylindrical fibres or spheres with cavities containing nanoscale samples, this study should open the way to nanoscale cross-sectional imaging using picosecond-ultrasonic computed tomography, allowing for the first time the $3 \mathrm{D}$ acoustic imaging of media with nanoscale resolution in both the lateral and indepth directions. Moreover, by the use of metal-coated cylindrical or spherical sub-micron hollowed regions on the end of an immersed tapered fibre in a standard near-field scanning optical microscope geometry, pump and probe light internal to the fibre can be used to follow focused $\mathrm{GHz}$ acoustic phonon pulses in liquids through Brillouin scattering, thus significantly extending the resolution of $3 \mathrm{D}$ acoustic imaging ${ }^{41}$ of biological structures to the nanoscale.

\section{CONFLICT OF INTEREST}

The authors declare no conflict of interest.

\section{AUTHOR CONTRIBUTIONS}

TD and OBW wrote the text. TD, KI and MT carried out the experiments. VEG, OM, OBW and TD performed the theoretical analysis. IAV, PHO and MT carried out the simulations. ST and MF prepared the sample. OBW and MT formulated the research plan. All authors reviewed the manuscript.

\section{ACKNOWLEDGEMENTS}

We acknowledge Grants-in-Aid for Scientific Research from the Ministry of Education, Culture, Sports, Science and Technology (MEXT), as well as support from the Japanese Society for the Promotion of Science (JSPS).

1 Huynh A, Lanzillotti-Kimura ND, Jusserand B, Perrin B, Fainstein A et al. Subterahertz phonon dynamics in acoustic nanocavities. Phys Rev Lett 2006; 97: 115502.

2 Berstermann T, Brüggemann C, Bombeck M, Akimov AV, Yakovlev DR et al. Optical bandpass switching by modulating a microcavity using ultrafast acoustics. Phys $\operatorname{Rev} B$ 2010; 81: 085316.

3 Tomes M, Carmon T. Photonic micro-electromechanical systems vibrating at X-band (11-GHz) rates. Phys Rev Lett 2009; 102: 113601.

4 Eichenfield M, Chan J, Camacho RM, Vahala KJ, Painter O. Optomechanical crystals. Nature 2009; 462: 78-82.

5 Kang MS, Brenn A, Russell P St J. All-optical control of gigahertz acoustic resonances by forward stimulated interpolarization scattering in a photonic crystal fiber. Phys Rev Lett 2010; 105: 153901.

6 Kelf TA, Tanaka Y, Matsuda O, Larsson EM, Sutherland DS et al. Ultrafast vibrations of gold nanorings. Nano Lett 2011; 11: 3893-3898.

7 Kelf TA, Hoshii W, Otsuka PH, Sakuma H, Veres IA et al. Mapping gigahertz vibrations in a plasmonic-phononic crystal. N J Phys 2013; 15: 023013.

8 Schumacher T, Kratzer K, Molnar D, Hentschel M, Giessen H et al. Nanoantennaenhanced ultrafast nonlinear spectroscopy of a single gold nanoparticle. Nat Commun 2011; 2: 333.

9 Lin K-H, Lai C-M, Pan C-C, Chyi J-I, Shi J-W et al. Spatial manipulation of nanoacoustic waves with nanoscale spot sizes. Nat Nanotechnol 2007; 2: 704-708.

10 Briggs A, Kolosov O. Acoustic Microscopy. Oxford, New York: Oxford University Press; 2009.

11 Daly BC, Holme NCR, Buma T, Branciard C, Norris TB et al. Imaging nanostructures with coherent phonon pulses. Appl Phys Lett 2004; 84: 5180-5182.

12 Zhang S, Péronne E, Belliard L, Vincent S, Perrin B. Three-dimensional acoustic wavefront imaging in anisotropic systems by picosecond acoustics. J Appl Phys 2011; 109: 033507.

13 Dehoux T, Kelf TA, Tomoda M, Matsuda O, Wright OB et al. Vibrations of microspheres probed with ultrashort optical pulses. Opt Lett 2009; 34: 3740-3742.

14 Ségur D, Guillet $Y$, Audoin B. Intrinsic geometric scattering probed by picosecond optoacoustics in a cylindrical cavity: application to acoustic and optical characterizations of a single micron carbon fiber. App/ Phys Lett 2010; 97: 031901.

15 Belliard L, Cornelius TW, Perrin B, Kacemi N, Becerra L et al. Vibrational response of free standing single copper nanowire through transient reflectivity microscopy. J Appl Phys 2013; 114: 193509.

16 Yu K, Zijlstra P, Sader JE, Xu Q-H, Orrit M et al. Damping of acoustic vibrations of immobilized single gold nanorods in different environments. Nano Lett 2013; 13: 2710-2716.

17 Mante P-A, Wu Y-C, Lin Y-T, Ho C-Y, Tu L-W et al. Gigahertz coherent guided acoustic phonons in AIN/GaN nanowire superlattices. Nano Lett 2013; 13: 1139-1144.

18 Jean C, Belliard L, Cornelius TW, Thomas O, Toimil-Molares ME et al. Direct observation of gigahertz coherent guided acoustic phonons in free-standing single copper nanowires. J Phys Chem Lett 2014; 5: 4100-4104.

19 Fujiwara M, Toubaru K, Takeuchi S. Optical transmittance degradation in tapered fibers. Opt Express 2011; 19: 8596-8601.

20 Anisimov SI, Kapeliovich BL, Perelman TL. Electron emission from metal surfaces exposed to ultrashort laser pulses. Zh Eksp Teor Fiz 1974; 66: 776-781.

21 Corkum PB, Brunel F, Sherman NK, Srinivasan-Rao T. Thermal response of metals to ultrashort-pulse laser excitation. Phys Rev Lett 1988; 61: 2886-2889.

22 Saito T, Matsuda O, Wright OB. Picosecond acoustic phonon pulse generation in nickel and chromium. Phys Rev B 2003; 67: 205421.

23 Wright OB. Ultrafast nonequilibrium stress generation in gold and silver. Phys Rev $B$ 1994; 49: 9985.

24 Lide D. CRC Handbook of Chemistry and Physics. Boca Raton: CRC Press. 1999. 
25 Thomsen C, Grahn HT, Maris HJ, Tauc J. Surface generation and detection of phonons by picosecond light pulses. Phys Rev B 1986; 34: 4129-4138.

26 Matsuda O, Larciprete MC, Voti RL, Wright OB. Fundamentals of picosecond laser ultrasonics. Ultrasonics 2015; 56: 3-20.

27 Ruello P, Gusev VE. Physical mechanisms of coherent acoustic phonons generation by ultrafast laser action. Ultrasonics 2015; 56: 21-35.

$28 \mathrm{Lin} \mathrm{H-N}$, Stoner RJ, Maris HJ, Tauc J. Phonon attenuation and velocity measurements in transparent materials by picosecond acoustic interferometry. J Appl Phys 1991; 69: 3816-3822.

29 Lomonosov AM, Ayouch A, Ruello P, Vaudel G, Baklanov MR et al. Nanoscale noncontact subsurface investigations of mechanical and optical properties of nanoporous low-k material thin film. ACS Nano 2012; 6: 1410-1415.

30 Viktorov IA. Rayleigh and Lamb Waves. New York: Plenum Press; 1967.

31 Tyc T. Gouy phase for full-aperture spherical and cylindrical waves. Opt Lett 2012; 37: 924-926.

32 Vacher R, Pelous J, Plicque F, Zarembowitch A. Ultrasonic and Brillouin scattering study of the elastic properties of vitreous silica between 10 and $300 \mathrm{~K}$. J Non-Cryst Solids 1981; 45: 397-410.

33 Bracewell RM. The Fourier Transform and its Applications. New York: McGraw-Hill; 1965.

34 Siegman AE. Lasers. California: University Science Books; 1986.

35 Laures P. Geometrical approach to Gaussian beam propagation. Appl Opt 1967; 6: 747-755.
36 Saleh BEA, Teich MC. Fundamentals of Photonics. New York: Wiley; 2007.

37 Tomoda M, Matsuda O, Wright OB, Voti RL. Tomographic reconstruction of acoustic strain propagation. App/ Phys Lett 2007; 90: 041114.

38 Mechri C, Ruello P, Breteau JM, Baklanov MR, Verdonck P et al. Depth-profiling of elastic inhomogeneities in transparent nanoporous low-k materials by picosecond ultrasonic interferometry. Appl Phys Lett 2009; 95: 091907.

39 Steigerwald A, Xu Y, Qi JQ, Gregory J, Liu XY et al. Semiconductor point defect concentration profiles measured using coherent acoustic phonon waves. App Phys Lett 2009; 94: 111910.

40 Kak AC, Slaney M. Principles of Computerized Tomographic Imaging. New York: IEEE Press; 1988

41 Danworaphong S, Tomoda M, Matsumoto Y, Matsuda O, Ohashi T et al. Threedimensional imaging of biological cells with picosecond ultrasonics. Appl Phys Lett 2015; 106: 163701 .

(c) (1) $\Theta$ This work is licensed under a Creative Commons AttributionBY NC ND NonCommercial-NoDerivs 4.0 International License. The images or other third party material in this article are included in the article's Creative Commons license, unless indicated otherwise in the credit line; if the material is not included under the Creative Commons license, users will need to obtain permission from the license holder to reproduce the material. To view a copy of this license, visit http:// creativecommons.org/licenses/by-nc-nd/4.0/

Supplementary Information for this article can be found on the Light: Science \& Applications' website (http://www.nature.com/lsa). 